PROCEEDINGS OF THE

AMERICAN MATHEMATICAL SOCIETY

Volume 125, Number 8, August 1997, Pages 2401-2407

S 0002-9939(97)04028-8

\title{
EXISTENCE OF BADE FUNCTIONALS FOR COMPLETE BOOLEAN ALGEBRAS OF PROJECTIONS IN FRÉCHET SPACES
}

\author{
W. J. RICKER
}

(Communicated by Palle E. T. Jorgensen)

\begin{abstract}
A classical result of W. Bade states that if $\mathcal{M}$ is any $\sigma-$ complete Boolean algebra of projections in an arbitrary Banach space $X$ then, for every $x_{0} \in X$, there exists an element $x^{\prime}$ (called a Bade functional for $x_{0}$ with respect to $\mathcal{M}$ ) in the dual space $X^{\prime}$, with the following two properties: (i) $M \mapsto\left\langle M x_{0}, x^{\prime}\right\rangle$ is non-negative on $\mathcal{M}$ and, (ii) $M x_{0}=0$ whenever $M \in \mathcal{M}$ satisfies $\left\langle M x_{0}, x^{\prime}\right\rangle=0$. It is shown that a Fréchet space $X$ has this property if and only if it does not contain an isomorphic copy of the sequence space $\omega=\mathbb{C}^{\mathbb{N}}$.
\end{abstract}

A Boolean algebra $\mathcal{M}$ of selfadjoint projections in a Hilbert space $H$ has the property that for every $x_{0} \in H$ the inner product $\left\langle E x_{0}, x_{0}\right\rangle$, with $E \in \mathcal{M}$, is nonnegative and vanishes only if $E x_{0}=0$. A satisfactory extension to the Banach space setting of this useful property of the inner product in Hilbert spaces is the following remarkable result of $\mathrm{W}$. Bade, [1, Theorem 3.1].

Theorem 1. Let $\mathcal{M}$ be a $\sigma$-complete Boolean algebra of projections in a Banach space $X$. Then, for each $x_{0} \in X$, there exists a continuous linear functional $x^{\prime} \in X^{\prime}$ (called a Bade functional for $x_{0}$ with respect to $\mathcal{M}$ ) with the properties

(i) $\left\langle M x_{0}, x^{\prime}\right\rangle \geq 0, \quad$ for all $M \in \mathcal{M}$, and

(ii) if $\left\langle M x_{0}, x^{\prime}\right\rangle=0$ for some $M \in \mathcal{M}$, then $M x_{0}=0$.

Theorem 1 fails to be true in the non-normable setting, even in Fréchet (locally convex) spaces. Indeed, the Boolean algebra $\mathcal{M}$ generated by the co-ordinate projections in the Fréchet space $\omega$ of all complex sequences, equipped with the topology of co-ordinatewise convergence, is $\sigma$-complete but the element $x_{0}=(1,1, \ldots)$ cannot have any Bade functional with respect to $\mathcal{M}$ since the continuous dual space $\omega^{\prime}$ consists of all complex sequences which have only finitely many non-zero terms.

Let $\mathcal{M}$ be a $\sigma$-complete Boolean algebra of projections in a locally convex Hausdorff space $X$. Then $\mathcal{M}$ is said to have Property- $(B)$ if, for every $x_{0} \in X$, there exists $x^{\prime} \in X^{\prime}$ satisfying (i) and (ii) of Theorem 1. The space $X$ is said to have the Bade property if every $\sigma$-complete Boolean algebra of projections in $X$ has Property-(B). Bade's classical theorem above asserts that every Banach space has the Bade property. The above example shows that Fréchet spaces in general do

Received by the editors March 4, 1996.

1991 Mathematics Subject Classification. Primary 47B15, 46G10, 47C05.

(C)1997 American Mathematical Society 
not have the Bade property; it turns out that this example is essentially a paradigm. The aim of this note is to establish the following result.

Theorem 2. Let $X$ be a Fréchet space. Then $X$ has the Bade property if and only if it does not contain an isomorphic copy of $\omega$.

\section{Preliminaries}

A Fréchet space $X$ is said to have Rybakov's property, [5], if for every $\sigma$-additive, $X$-valued vector measure $\nu$ (defined on a $\sigma$-algebra of sets $\Sigma$ ) there exists $x^{\prime} \in X^{\prime}$ such that $\nu \ll\left|\left\langle\nu, x^{\prime}\right\rangle\right|$ (i.e. $\nu(F)=0$ for every measurable set $F \subseteq E$ whenever $\left.\left|\left\langle\nu, x^{\prime}\right\rangle\right|(E)=0\right)$. Here $\left\langle\nu, x^{\prime}\right\rangle$ denotes the complex measure $E \mapsto\left\langle\nu(E), x^{\prime}\right\rangle$ and $\left|\left\langle\nu, x^{\prime}\right\rangle\right|$ denotes the total variation measure of $\left\langle\nu, x^{\prime}\right\rangle$.

Proposition 1 ([5, Theorem 2.2]). For a real Fréchet space $X$ the following conditions are equivalent.

(i) X admits a continuous norm.

(ii) Every convex, weakly compact subset of $X$ is the closed convex hull of its exposed points.

(iii) $X$ has Rybakov's property.

We will require the following extension of this result to Fréchet spaces over $\mathbb{C}$.

Proposition 2. For a Fréchet space $X$ the following conditions are equivalent.

(i) $X$ contains no isomorphic copy of $\omega$.

(ii) $X$ admits a continuous norm.

(iii) $X$ has Rybakov's property.

Proof. The equivalence of (i) and (ii) is well known; see [2] or [6, Theorem 7.2.7], for example.

(ii) $\Longrightarrow$ (iii). Let $X_{\mathbb{R}}$ denote $X$ considered as a linear space over $\mathbb{R}$, in which case $X_{\mathbb{R}}$ also admits a continuous norm (the same one that $X$ does). Let $\nu: \Sigma \longrightarrow X$ be a vector measure, meaning that $\nu\left(A_{n}\right) \longrightarrow 0$ in $X$ whenever $A_{n} \downarrow \phi$ in $\Sigma$. But then also $\nu\left(A_{n}\right) \longrightarrow 0$ in $X_{\mathbb{R}}$ and so $\nu$ is still a vector measure when considered as taking its values in $X_{\mathbb{R}}$. By Proposition 1 there exists a continuous linear functional $x^{\prime}: X_{\mathbb{R}} \longrightarrow \mathbb{R}$ such that $\nu \ll\left|\left\langle\nu, x^{\prime}\right\rangle\right|$. Define $\xi^{\prime}: X \longrightarrow \mathbb{C}$ by

$$
\left\langle x, \xi^{\prime}\right\rangle=\left\langle x, x^{\prime}\right\rangle-i\left\langle i x, x^{\prime}\right\rangle, \quad x \in X,
$$

in which case $\xi^{\prime} \in X^{\prime}$ and satisfies

$$
\left\langle\nu(E), \xi^{\prime}\right\rangle=\left\langle\nu(E), x^{\prime}\right\rangle-i\left\langle i \nu(E), x^{\prime}\right\rangle, \quad E \in \Sigma .
$$

Accordingly, if $E \in \Sigma$ is a set such that $\left\langle\nu(F), \xi^{\prime}\right\rangle=0$ for all $F \in \Sigma$ with $F \subseteq$ $E$, then also $\operatorname{Re}\left(\left\langle\nu(F), \xi^{\prime}\right\rangle\right)=\left\langle\nu(F), x^{\prime}\right\rangle=0$ for all $F \in \Sigma$ with $F \subseteq E$. That is, $\left|\left\langle\nu, x^{\prime}\right\rangle\right|(E)=0$ whenever $\left|\left\langle\nu, \xi^{\prime}\right\rangle\right|(E)=0$ showing that $\left|\left\langle\nu, x^{\prime}\right\rangle\right| \ll\left|\left\langle\nu, \xi^{\prime}\right\rangle\right|$. Accordingly, $\nu \ll\left|\left\langle\nu, \xi^{\prime}\right\rangle\right|$. Since $\nu$ is an arbitrary $X$-valued measure it follows that $X$ has Rybakov's property.

(iii) $\Longrightarrow$ (ii). We show that $X_{\mathbb{R}}$ has Rybakov's property whenever $X$ does; the conclusion then follows from Proposition 1 since any continuous norm $\varphi$ on $X_{\mathbb{R}}$ induces a continuous norm $\tilde{\varphi}$ on $X$ via the formula $\tilde{\varphi}(x)=\sup \{\varphi(\alpha x): \alpha \in \mathbb{C},|\alpha|=$ $1\}$ for $x \in X$. So, let $\nu: \Sigma \longrightarrow X_{\mathbb{R}}$ be a vector measure. By a similar argument as in the proof of (ii) $\Longrightarrow$ (iii) it follows that $\nu$ is also a vector measure when considered as being $X$-valued. By assumption there is $x^{\prime} \in X^{\prime}$ satisfying $\nu \ll\left|\left\langle\nu, x^{\prime}\right\rangle\right|$. Define 
elements $\xi_{1}^{\prime}, \xi_{2}^{\prime} \in X_{\mathbb{R}}^{\prime}$ by $\left\langle x, \xi_{1}^{\prime}\right\rangle=\operatorname{Re}\left(\left\langle x, x^{\prime}\right\rangle\right)$ and $\left\langle x, \xi_{2}^{\prime}\right\rangle=\operatorname{Im}\left(\left\langle x, x^{\prime}\right\rangle\right)$, for each $x \in X_{\mathbb{R}}$. Then $\mu_{j}=\left\langle\nu, \xi_{j}^{\prime}\right\rangle: \Sigma \longrightarrow \mathbb{R}$ is a signed measure, for each $j \in\{1,2\}$. By [3, IX Lemma 2.1] there exists $t \in(0,1)$ such that

$$
\mu_{j} \ll\left|t \mu_{1}+(1-t) \mu_{2}\right|=\left|\left\langle\nu, z^{\prime}\right\rangle\right|, \quad j \in\{1,2\},
$$

where $z^{\prime}=t \xi_{1}^{\prime}+(1-t) \xi_{2}^{\prime}$ is then an element of $X_{\mathbb{R}}^{\prime}$. Suppose that $\left|\left\langle\nu, z^{\prime}\right\rangle\right|(E)=0$, that is, $E$ is $\mu_{j}$-null, for each $j \in\{1,2\}$. This is equivalent to $\mu_{j}(F)=\left\langle\nu(F), \xi_{j}^{\prime}\right\rangle=0$ for every $j \in\{1,2\}$ and $F \in \Sigma$ with $F \subseteq E$. That is, $\operatorname{Re}\left(\left\langle\nu(F), x^{\prime}\right\rangle\right)=0=$ $\operatorname{Im}\left(\left\langle\nu(F), x^{\prime}\right\rangle\right)$, for all $F \in \Sigma$ with $F \subseteq E$, which is equivalent to $\left\langle\nu(F), x^{\prime}\right\rangle=0$, for all $F \in \Sigma$ with $F \subseteq E$. That is, $\left|\left\langle\nu, x^{\prime}\right\rangle\right|(E)=0$. So, we have shown that $\left|\left\langle\nu, x^{\prime}\right\rangle\right| \ll\left|\left\langle\nu, z^{\prime}\right\rangle\right|$ and hence, $\nu \ll\left|\left\langle\nu, z^{\prime}\right\rangle\right|$. Since $z^{\prime} \in X_{\mathbb{R}}^{\prime}$ it follows that $X_{\mathbb{R}}$ has Rybakov's property.

Combining Proposition 2 with Theorem 2 (yet to be proved) gives the following consequence.

Corollary 2.1. For a Fréchet space $X$ the following conditions are equivalent.

(i) $X$ does not contain an isomorphic copy of $\omega$.

(ii) $X$ admits a continuous norm.

(iii) $X$ has Rybakov's property.

(iv) $X$ has the Bade property.

The space of all continuous linear operators of a locally convex Hausdorff space $X$ into itself is denoted by $L(X)$. A Boolean algebra of projections $\mathcal{M} \subseteq L(X)$, with unit always the identity operator $I$ in $X$, is said to be complete ( $\sigma$-complete), in the sense of Bade, if it is complete ( $\sigma$ - complete) as an abstract Boolean algebra and, whenever $\left\{M_{\alpha}\right\}_{\alpha}$ is a family (sequence) from $\mathcal{M}$, then

$$
\left(\wedge_{\alpha} M_{\alpha}\right) X=\cap_{\alpha} M_{\alpha} X \quad \text { and } \quad\left(v_{\alpha} M_{\alpha}\right) X=\overline{\mathrm{sp}}\left\{\cup_{\alpha} M_{\alpha} X\right\} .
$$

Of course, the partial order in $\mathcal{M}$ is range inclusion, i.e. $M \leq N$ if $M X \subseteq N X$. If $X$ is a Fréchet space, then $\mathcal{M}$ is necessarily an equicontinuous part of $L(X)$, [9, Proposition 1.2]. Moreover, it is known that $\mathcal{M}=P(\Sigma)$ is the range of some spectral measure $P: \Sigma \longrightarrow L(X)$ defined on a $\sigma$-algebra of sets $\Sigma$ of some set $\Omega$, $[9$, p. 299]. By a spectral measure we mean that $P$ is $\sigma$-additive with respect to the strong operator topology in $L(X)$, that $P(\Omega)=I$, and that $P$ is multiplicative (i.e. $P(E \cap F)=P(E) P(F)$ for all $E, F \in \Sigma)$.

Given a vector $x_{0} \in X$ the cyclic space $\mathcal{M}\left[x_{0}\right]$ generated by $x_{0}$ with respect to $\mathcal{M}$ is the closure of the linear span of $\left\{M x_{0} ; M \in \mathcal{M}\right\}$. The $X$-valued (actually $\mathcal{M}\left[x_{0}\right]$-valued) vector measure $P x_{0}: \Sigma \longrightarrow X$ is defined by $P x_{0}: E \mapsto P(E) x_{0}$, for each $E \in \Sigma$. Associated with the vector measure $P x_{0}$ is its (locally convex Hausdorff) space $L^{1}\left(P x_{0}\right)$ of (equivalence classes of) $P x_{0}$-integrable functions $f$ : $\Omega \longrightarrow \mathbb{C}$, equipped with the topology of convergence in mean; see [4, Section 1], for example. For the notion of a closed vector measure we also refer to [4, Section 1]. The integration map $I_{P x_{0}}: L^{1}\left(P x_{0}\right) \longrightarrow X$ is defined by $f \mapsto \int_{\Omega} f d P x_{0}$, for each $f \in L^{1}\left(P x_{0}\right)$.

Proposition 3 ([4, Proposition 2.1]). Let $X$ be a quasicomplete locally convex Hausdorff space such that $L(X)$ is sequentially complete for the strong operator topology. Let $\mathcal{M} \subseteq L(X)$ be an equicontinuous, complete Boolean algebra of projections, displayed as the range of a closed, equicontinuous spectral measure $P: \Sigma \longrightarrow$ 
$L(X)$. Then, for each $x_{0} \in X$, the integration map $I_{P x_{0}}: L^{1}\left(P x_{0}\right) \longrightarrow \mathcal{M}\left[x_{0}\right]$ induces on the cyclic space $\mathcal{M}\left[x_{0}\right]$ the structure of a Dedekind complete, locally solid (complex) Riesz space with Lebesgue topology in which $x_{0}$ is a weak order unit. The absolute value of an element $\int_{\Omega} f d P x_{0} \in \mathcal{M}\left[x_{0}\right]$ is the element $\int_{\Omega}|f| d P x_{0} \in \mathcal{M}\left[x_{0}\right]$. Moreover, $I_{P x_{0}}$ is a Riesz isomorphism as well as a topological isomorphism and the absolute value mapping on $\mathcal{M}\left[x_{0}\right]$ is continuous.

\section{Proof of Theorem 2}

Let $\mathcal{M} \subseteq L(X)$ be any $\sigma$-complete Boolean algebra of projections. Since $\mathcal{M}$ is equicontinuous, [9, Proposition 1.2], the closure $\overline{\mathcal{M}}$ of $\mathcal{M}$ in $L(X)$, with respect to the strong operator topology, is an equicontinuous complete Boolean algebra, $[9$, Proposition 3.17]. Since $\mathcal{M}\left[x_{0}\right]=\overline{\mathcal{M}}\left[x_{0}\right]$ it is clear from the definition of the Bade property that it suffices to show that every complete Boolean algebra of projections in $L(X)$ has Property-(B) if and only if $X$ does not contain an isomorphic copy of $\omega$.

Suppose that $X$ does not contain a copy of $\omega$ in which case it admits a continuous norm (by Proposition 2). Let $\mathcal{M} \subseteq L(X)$ be any complete Boolean algebra, necessarily equicontinuous. Fix $x_{0} \in X$. Let $P: \Sigma \longrightarrow L(X)$ be a closed, equicontinuous spectral measure such that $\mathcal{M}=P(\Sigma),[9, \mathrm{p} .299]$. Since $X$ admits a continuous norm so does $\mathcal{M}\left[x_{0}\right]$. So, to establish that $X$ has the Bade property it suffices to establish the following result.

Proposition 4. Let $X$ be a quasicomplete locally convex Hausdorff space such that $L(X)$ is sequentially complete for the strong operator topology. Let $\mathcal{M} \subseteq L(X)$ be a complete, equicontinuous Boolean algebra of projections. Suppose $x_{0} \in X$ has the property that the cyclic space $\mathcal{M}\left[x_{0}\right]$ is a Fréchet space for the relative topology from $X$. The following statements are equivalent.

(i) $x_{0}$ has a Bade functional with respect to $\mathcal{M}$.

(ii) $\mathcal{M}\left[x_{0}\right]$ admits a continuous norm.

Proof. (i) $\Longrightarrow$ (ii) Suppose that $x^{\prime} \in X^{\prime}$ is a Bade functional for $x_{0}$. By Proposition 3 the restriction of $x^{\prime}$ to $\mathcal{M}\left[x_{0}\right]$ acts via the formula

$$
\left\langle\int_{\Omega} f d P x_{0}, x^{\prime}\right\rangle=\int_{\Omega} f d\left\langle P x_{0}, x^{\prime}\right\rangle, \quad f \in L^{1}\left(P x_{0}\right),
$$

where $P: \Sigma \longrightarrow L(X)$ is a closed, equicontinuous spectral measure such that $\mathcal{M}=P(\Sigma)$. Define $q: \mathcal{M}\left[x_{0}\right] \longrightarrow[0, \infty)$ by $q\left(\int_{\Omega} f d P x_{0}\right)=\int_{\Omega}|f| d\left\langle P x_{0}, x^{\prime}\right\rangle$, for each $f \in L^{1}\left(P x_{0}\right)$. By property (i) of Bade functionals (cf. Theorem 1) it is clear that $q$ does indeed take non-negative values and, by the properties of integration with respect to a non-negative measure, it is clear that $q$ is a seminorm on $\mathcal{M}\left[x_{0}\right]$. Since

$$
\int_{\Omega}|f| d\left\langle P x_{0}, x^{\prime}\right\rangle=\left\langle\int_{\Omega}|f| d P x_{0}, x^{\prime}\right\rangle=\left\langle\left|\int_{\Omega} f d P x_{0}\right|_{\mathcal{M}\left[x_{0}\right]}, x^{\prime}\right\rangle,
$$

for each $f \in L^{1}\left(P x_{0}\right)$, where $|\cdot|_{\mathcal{M}\left[x_{0}\right]}$ is the absolute value in the Riesz space $\mathcal{M}\left[x_{0}\right]$, necessarily continuous (cf. Proposition 3 ), it follows that $q$ is continuous on $\mathcal{M}\left[x_{0}\right]$. Finally, to see that $q$ is actually a norm, suppose that $q\left(\int_{\Omega} f d P x_{0}\right)=0$, i.e. $|f|$ is $\left\langle P x_{0}, x^{\prime}\right\rangle-$ null. By property (ii) of Bade functionals (cf. Theorem 1) and the fact that $\mathcal{M}=P(\Sigma)$, there is a set $E \in \Sigma$ such that $f(w)=0$ for $w \notin E$ and $P(E) x_{0}=0$. By multiplicativity of $P$ it follows that $P(F) x_{0}=0$ for every $F \in \Sigma$ 
such that $F \subseteq E$ i.e. $E$ is a $P x_{0}-$ null set and so $f$ is $P x_{0}-$ null. Accordingly, $\int_{\Omega} f d P x_{0}=0$ which establishes that $q$ is a norm.

(ii) $\Longrightarrow$ (i) Suppose that $\mathcal{M}\left[x_{0}\right]$ admits a continuous norm. By the Rybakov property for the vector measure $P x_{0}: \Sigma \longrightarrow \mathcal{M}\left[x_{0}\right]$ (cf. Proposition 2) there exists $x^{\prime} \in \mathcal{M}\left[x_{0}\right]^{\prime}$ (which can also be considered as an element of $X^{\prime}$ by the Hahn-Banach theorem) satisfying $P x_{0} \ll\left|\left\langle P x_{0}, x^{\prime}\right\rangle\right|$. By the Radon-Nikodym theorem there exists a $\Sigma$-measurable function $\varphi: \Omega \longrightarrow \mathbb{C}$ with $|\varphi(w)|=1$, for $\left\langle P x_{0}, x^{\prime}\right\rangle$ - a.e. point $w \in \Omega$, such that

$$
\left|\left\langle P x_{0}, x^{\prime}\right\rangle\right|(E)=\int_{E} \varphi d\left\langle P x_{0}, x^{\prime}\right\rangle, \quad E \in \Sigma .
$$

Define $\varphi$ to be zero on the $\left\langle P x_{0}, x^{\prime}\right\rangle$-null set for which $|\varphi| \neq 1$, in which case $\varphi$ is a bounded, $\Sigma$-measurable function. Accordingly, $\varphi$ is $P$-integrable and $T=$ $\int_{\Omega} \varphi d P \in L(X),\left[9\right.$, p.300]. Let $z^{\prime}=T^{\prime} x^{\prime}$ where $T^{\prime}$ denotes the dual operator to $T$. Then $z^{\prime} \in X^{\prime}$ satisfies

$$
\left\langle P(E) x_{0}, z^{\prime}\right\rangle=\int_{E} \varphi d\left\langle P x_{0}, x^{\prime}\right\rangle=\left|\left\langle P x_{0}, x^{\prime}\right\rangle\right|(E), \quad E \in \Sigma .
$$

It follows that $\left\langle P x_{0}, z^{\prime}\right\rangle$ is a non-negative measure with the property that $P(E) x_{0}=$ 0 whenever $\left\langle P(E) x_{0}, z^{\prime}\right\rangle=0$, i.e. $z^{\prime}$ is a Bade functional for $x_{0}$ with respect to $\mathcal{M}$.

Conversely, suppose that $X$ does contain an isomorphic copy of $\omega$. Then it contains a complemented copy of $\omega,[6$, Theorem 7.2.7], and so we have the direct decomposition $X=Y \oplus \omega$. Let $\Sigma=2^{\mathbb{N}}$ and define a spectral measure $Q: \Sigma \longrightarrow L(\omega)$ by $Q(E): \psi \mapsto \chi_{E} \psi$ (co-ordinatewise multiplication), for each $\psi \in \omega$ and $E \in \Sigma$. Then define a spectral measure $P: \Sigma \longrightarrow L(X)$ by $P(E)=0_{Y} \oplus Q(E)$, for each $E \in \Sigma$, where $0_{Y}$ is the zero operator on $Y$. As noted in the introduction the vector $u_{0}=(1,1, \ldots)$ of $\omega$ has no Bade functional with respect to $Q(\Sigma)$ from which it follows easily ( since $X^{\prime}=Y^{\prime} \oplus \omega^{\prime}$ ) that $x_{0}=0 \oplus u_{0}$ is an element of $X$ which has no Bade functional with respect to the complete Boolean algebra $\mathcal{M}=P(\Sigma) \subseteq L(X)$. Accordingly, $X$ does not have the Bade property. Equivalently, if $X$ has the Bade property, then it cannot contain a copy of $\omega$. The proof of Theorem 2 is thereby complete.

Remark 1. (i) Since $\mathcal{M}\left[x_{0}\right]$ is a Banach space whenever $X$ is a Banach space, it follows that Proposition 4 applies in a Banach space $X$ to every $x_{0} \in X$. Accordingly, Proposition 4 contains the classical Bade functional theorem. An examination of the proof of (ii) $\Longrightarrow$ (i) in Proposition 4 shows that it is quite a different proof (perhaps even more transparent) than W. Bade's original proof given in [1, Theorem 3.1]. Of course, it is based on Rybakov's theorem which, even in the Banach space setting, was not available until 1970; see [8].

(ii) The proof of (i) $\Longrightarrow$ (ii) in Proposition 4 applies in general; it does not require $\mathcal{M}\left[x_{0}\right]$ to be a Fréchet space. This was noted in $[9, \mathrm{p} .315]$, with a slightly different proof (based on order properties rather than vector integration), where the full statement of Proposition 4 is also alluded to, but without a proof. Whatever proof the author had in mind there it could not have been that given here since, again, Rybakov's theorem was not available at that time.

A Boolean algebra of projections $\mathcal{M}$ acting in a locally convex Hausdorff space $X$ is said to be cyclic if there exists $x_{0} \in X$ such that $X=\mathcal{M}\left[x_{0}\right]$. 
Proposition 5. Let $X$ be a Fréchet space which does not contain a copy of $\omega$ and let $\mathcal{M} \subseteq L(X)$ be a cyclic, $\sigma$-complete Boolean algebra of projections. Then there exists a continuous norm, $\|\cdot\|$, on $X$ such that $\mathcal{M} \subseteq L\left(X_{\|\cdot\|}\right)$, where $X_{\|\cdot\|}$ denotes $X$ equipped with the norm topology induced by $\|\cdot\|$. Moreover, $\|M\| \leq 1$ for every $M \in \mathcal{M}$, and $\mathcal{M}$ is $\sigma$-complete considered as a Boolean algebra in $L\left(X_{\|\cdot\|}\right)$.

Proof. Let $P: \Sigma \longrightarrow L(X)$ be a spectral measure such that $\mathcal{M}=P(\Sigma)$. By hypothesis there exists $x_{0} \in X$ such that $X=\mathcal{M}\left[x_{0}\right]$. By Proposition 3 the integration map $I_{P x_{0}}: L^{1}\left(P x_{0}\right) \longrightarrow X$ is a Riesz space and bicontinuous topological isomorphism which induces the absolute value map given by $|y|=\int_{\Omega}|f| d P x_{0}$ on $X$ if $y=\int_{\Omega} f d P x_{0}$. Let $x^{\prime} \in X^{\prime}$ be a Bade functional for $x_{0}$ with respect to $\mathcal{M}$. It was seen in the proof of Proposition 4 that

$$
\left\|I_{P x_{0}}(f)\right\|=\int_{\Omega}|f| d\left\langle P x_{0}, x^{\prime}\right\rangle, \quad f \in L^{1}\left(P x_{0}\right),
$$

is then a continuous norm on $X$.

Fix $M=P(E)$ in $\mathcal{M}$. Given $x=I_{P x_{0}}(f)$ in $X$, for some unique $f \in L^{1}\left(P x_{0}\right)$, it follows from basic properties of spectral integrals (cf. [4]) that

$$
M x=P(E) x=P(E) \int_{\Omega} f d P x_{0}=\int_{\Omega} f \chi_{E} d P x_{0}=I_{P x_{0}}\left(f \chi_{E}\right) .
$$

These equalities show that

$$
\|M x\|=\left\|I_{P x_{0}}\left(f \chi_{E}\right)\right\|=\int_{\Omega}\left|f \chi_{E}\right| d\left\langle P x_{0}, x^{\prime}\right\rangle \leq \int_{\Omega}|f| d\left\langle P x_{0}, x^{\prime}\right\rangle=\|x\| .
$$

That is, $\|M\| \leq 1$ and, in particular, $M \in L\left(X_{\|\cdot\|}\right)$.

To show that $\mathcal{M}$ is a $\sigma$-complete Boolean algebra when considered in $L\left(X_{\|\cdot\|}\right)$ it suffices to check that $P: \Sigma \longrightarrow L\left(X_{\|\cdot\|}\right)$ is $\sigma$-additive for the strong operator topology. But, suppose that $\left\{A_{n}\right\}_{n=1}^{\infty} \subseteq \Sigma$ is a sequence decreasing to $\phi$. Fix $x \in X$. Then $P\left(A_{n}\right) x \longrightarrow 0$, as $n \longrightarrow \infty$, in the topology of $X$. Since $\|\cdot\|: X \longrightarrow[0, \infty)$ is continuous also $\left\|P\left(A_{n}\right) x\right\| \longrightarrow 0$, as $n \longrightarrow \infty$. This establishes that $P$ is $\sigma$-additive in $L\left(X_{\|\cdot\|}\right)$ for the strong operator topology.

Proposition 6. Let $X$ be the strict inductive limit of an increasing sequence of Fréchet spaces $\left\{X_{n}\right\}_{n=1}^{\infty}$ such that no $X_{n}, n=1,2, \ldots$, contains an isomorphic copy of $\omega$. Then every equicontinuous, $\sigma$-complete Boolean algebra of projections $\mathcal{M} \subseteq L(X)$ has Property- $(B)$.

Proof. Fix $x_{0} \in X$. The equicontinuity of $\mathcal{M}$ implies that $B\left(x_{0}\right)=\left\{M x_{0} ; M \in \mathcal{M}\right\}$ is a bounded set in $X$. Accordingly, there exists a positive integer $n$ such that $B\left(x_{0}\right) \subseteq X_{n},\left[7\right.$, p.223]. Since the relative topology induced on $X_{n}$ by $X$ is precisely the given Fréchet space topology of $X_{n},[7$, p.222], it follows that the closure of the linear span of $B\left(x_{0}\right)$ taken in $X_{n}$ with respect to the metric topology coincides with $\mathcal{M}\left[x_{0}\right]$ formed in $X$. The conclusion now follows from Proposition 4.

Remark 2. Let $X$ be as in the statement of Proposition 6. Then every spectral measure $P: \Sigma \longrightarrow L(X)$ has Property-(B). This is immediate from Proposition 6 since the barrelledness of $X,[7, \mathrm{p} .368]$, and the boundedness of $\mathcal{M}=P(\Sigma)$ in $L(X),[9$, p.300], imply that the $\sigma$-complete Boolean algebra $\mathcal{M}$ is necessarily equicontinuous.

The final result, which is a simple consequence of Theorem 2, shows that there also exist extensive classes of non-metrizable lcH-spaces with the Bade property. 
Proposition 7. Let $Y$ be a Fréchet space and $X$ denote $Y$ equipped with its weak topology $\sigma\left(Y, Y^{\prime}\right)$. Then $X$ has the Bade property iff $Y$ does not contain a copy of $\omega$.

Proof. Since $Y$ has its Mackey topology, [7, p.263], it is known that $L(X)=L(Y)$ as vector spaces, [7, p.262]. Moreover, since the closure of a linear subspace of $Y$ with respect to the metric (= Mackey) topology coincides with the $\sigma\left(Y, Y^{\prime}\right)$-closure, it follows from the definition that a Boolean algebra of projections $\mathcal{M}$ is $\sigma$-complete considered as a part of $L(X)$ iff it is $\sigma$-complete considered as a part of $L(Y)$. Using these observations and the fact that $X^{\prime}=Y^{\prime}$ it is routine to check that $Y$ has the Bade property iff $X$ has the Bade property. The desired conclusion then follows from Theorem 2 .

\section{REFERENCES}

1. Bade, W., On Boolean algebras of projections and algebras of operators, Trans. Amer. Math. Soc. 80 (1955), 345-360. MR 17:513d

2. Bessaga, C. and Pelzynski A.A., On a class of $B_{0}$-spaces, Bull. Acad. Polon. Sci. 5 (1957), 375-377. MR 19:562b

3. Diestel, J. and Uhl. J.J. Jnr, Vector measures, Math. Surveys No.15, Amer. Math. Soc., Providence, 1977. MR 56:12216

4. Dodds, P. G., de Pagter, B. and Ricker, W.J., Reflexivity and order properties of scalar-type spectral operators in locally convex spaces, Trans. Amer. Math. Soc. 293 (1986), 355-380. MR 87d:47046

5. Fernández, A. and Naranjo, F., Rybakov's theorem for vector measures in Fréchet spaces, Indag. Math. (New Series), to appear.

6. Jarchow, H., Locally convex spaces, Stuttgart, Teubner, 1981. MR 83h:46008

7. Köthe, G., Topological vector spaces I, Springer-Verlag, Heidelberg, 1969.

8. Rybakov, V.I., Theorem of Bartle, Dunford and Schwartz on vector-valued measures, Mat. Zametik, 7 (1970), 247-254 (= Math. Notes 7, 147-151). MR 41:5591

9. Walsh, B., Structure of spectral measures on locally convex spaces, Trans. Amer. Math. Soc. 120 (1965), 295-326. MR 33:4690

School of Mathematics, University of New South Wales, Sydney, New South Wales, 2052 Australia 